\title{
Experimental validation of k-Wave: Nonlinear wave propagation in layered, absorbing fluid media
}

\author{
Eleanor Martin, Jiri Jaros and Bradley Treeby
}

\begin{abstract}
Models of ultrasound propagation in biologically relevant media have applications in planning and verification of ultrasound therapies and computational dosimetry. To be effective, the models must be able to accurately predict both the spatial distribution and amplitude of the acoustic pressure. This requires that the models are validated in absolute terms, which for arbitrarily heterogeneous media should be performed by comparison with measurements of the acoustic field. In this study, simulations performed using the open-source k-Wave acoustics toolbox, with a measurement-based source definition, were quantitatively validated against measurements of acoustic pressure in water and layered absorbing fluid media. In water, the measured and simulated spatial peak pressures agreed to within $3 \%$ under linear conditions and 7\% under non-linear conditions. After propagation through a planar or wedge shaped glycerolfilled phantom, the difference in spatial peak pressure was $8.5 \%$ and $10.7 \%$, respectively. These differences are within or close to the expected uncertainty of the acoustic pressure measurement. The $-6 \mathrm{~dB}$ width and length of the focus agreed to within $4 \%$ in all cases, and the focal positions were within $0.7 \mathrm{~mm}$ for the planar phantom and $1.2 \mathrm{~mm}$ for the wedge shaped phantom. These results demonstrate that when the acoustic medium properties and geometry are well known, accurate quantitative predictions of the acoustic field can be made using k-Wave.
\end{abstract}

\section{INTRODUCTION}

$\mathbf{M}$ ODELS of ultrasound propagation in biological media have a number of applications in therapeutic ultrasound including treatment planning and verification, patient selection, and computational dosimetry [1-3]. In these applications, patient safety and the effectiveness of treatments is critical, so it is important that the models used are able to accurately predict both the spatial distribution and absolute value of the acoustic pressure. To test their accuracy, models of ultrasound propagation should be validated under conditions that are analogous to the final conditions of use. This validation can be performed by comparison with analytical solutions, with other numerical models, or with measurements of acoustic pressure.

Manuscript received XX; revised XX. This work was supported in part by the Engineering and Physical Sciences Research Council (EPSRC), UK, grant numbers EP/P008860/1 and EP/L020262/1, and in part by European Union's Horizon 2020 research and innovation programme H2020 ICT 20162017 under grant agreement No 732411 (as an initiative of the Photonics Public Private Partnership), and in part by The Ministry of Education, Youth and Sports from the National Programme of Sustainability (NPU II) project IT4Innovations excellence in science - LQ1602 and by the IT4Innovations infrastructure which is supported from the Large Infrastructures for Research, Experimental Development and Innovations project IT4Innovations National Supercomputing Center - LM2015070.

E Martin and B Treeby are with the Department of Medical Physics and Biomedical Engineering, University College London, London, UK (email: elly.martin@ucl.ac.uk). J Jaros is with the Centre of Excellence IT4Innovations, Faculty of Information Technology, Brno University of Technology, Brno, CZ.
Analytical solutions are available for simple problems such as planar and curved uniform radiators in homogenous lossless media, and for a point source or plane wave with simple inhomogeneous medium geometries such as a half space, cylinder or sphere [4]. For other arbitrary geometries, and for absorbing media and nonlinear fields, comparison against other suitable models is a useful step (e.g. [5, 6]), but experimental validation is generally required to determine absolute accuracy.

One model that is increasingly being used for modelling therapeutic ultrasound fields is the open-source $\mathrm{k}$-Wave toolbox $[7,8]$. This solves a system of first-order coupled equations (equivalent to a generalised Westervelt equation) that accounts for nonlinear wave propagation in heterogeneous media with acoustic absorption that follows a frequency power law. The model equations are solved using a k-space pseudospectral method which minimises numerical dispersion [9]. To give a small number of examples, $\mathrm{k}$-Wave has recently been used by the international community to model high-intensity focused ultrasound [10], non-thermal ablation [11], neuromodulation [12], and opening the blood-brain barrier [13].

To date, both qualitative and quantitative experimental validations of $\mathrm{k}$-Wave have been performed under a limited range of conditions (see Table I). However, quantitative validation of nonlinear wave propagation in layered absorbing media has not yet been reported. Experimental validations of other models of ultrasound propagation in layered fluid media, such as those based on the Khokhlov-Zabolotskaya-Kuznetsov (KZK) equation and extended angular spectrum approach (ASA) are also presented in the literature (a number of representative examples are summarised in Table I). However, in general, neither the models or the data are freely available for the validation of other models.

In the current work, a quantitative experimental validation of $\mathrm{k}$-Wave is performed in water using a measurement-based source definition under weakly nonlinear conditions and with absorbing fluid phantoms immersed in water. Such validations are critical to establish the conditions under which k-Wave can be used to make accurate predictions of therapeutic ultrasound fields. In the approach taken here, the best estimate of the source and medium properties and geometry obtained from measurements were used for simulations. No iteration of these quantities was performed (e.g. to minimise differences between measurement and simulations), as to do so could mask errors in the model and measurement. This comparison which includes uncertainties in the measurement of pressure, material properties, and geometry, is representative of a 'real world' application of modelling of acoustic fields, for example, where the acoustic medium is defined using medical images 
and the source is defined by measurement.

\section{EXPERIMENTAL METHODS}

\section{A. Experimental setup}

An acoustic field was generated by a single element spherically focusing transducer with a nominal aperture diameter of $64 \mathrm{~mm}$ and radius of curvature of $98 \mathrm{~mm}$ (H151, Sonic Concepts, Bothell, WA, USA). For all measurements, the transducer was driven at a frequency of $1.1 \mathrm{MHz}$ with a 4-cycle burst generated by an arbitrary waveform generator (33522A, Agilent, Berkshire, UK), amplified by $75 \mathrm{~W}$ RF power amplifier (A075, Electronics and Innovation Ltd., Rochester, NY, USA), and coupled to the transducer via an electrical impedance matching network. The drive voltage was monitored at the input to the impedance matching network using an oscilloscope probe (Tektronix TPP0850) and digital phosphor oscilloscope (DPO5034B, Tektronix UK Ltd., Berkshire, UK). The transducer was mounted in an automated scanning tank filled with degassed, deionised water and the acoustic fields were measured with a calibrated $0.2 \mathrm{~mm}$ PVDF needle hydrophone (Precision Acoustics, Dorchester, UK) positioned with a 3-axis $(X, Y, Z)$ computer-controlled translation stage. The complex frequency response of the hydrophone was obtained by calibration at the National Physical Laboratory in the range $0.1-40 \mathrm{MHz}$. The $-6 \mathrm{~dB}$ bandwidth of the hydrophone was $35 \mathrm{MHz}$. Waveforms were acquired, digitised and stored via the digital phosphor oscilloscope, under the control of the scanning tank software, with a sample rate of $100 \mathrm{MHz}$ and 32 averages. Signals were acquired in a time window that included the earliest and latest arrival times of the pulse at the measurement position. To obtain the acoustic pressure from the acquired voltage waveforms, a Tukey window was applied before bandpass filtering ( $-6 \mathrm{~dB}$ pass band: $200 \mathrm{kHz}-10$ $\mathrm{MHz}$ ) and deconvolution of the complex frequency response of the hydrophone. During measurements, the water temperature was monitored with a USB thermocouple (US-TC01 + J type thermocouple, National Instruments Ltd., Newbury, UK) and was maintained at $20.6 \pm 0.3{ }^{\circ} \mathrm{C}$.

\section{B. Source characterisation and free-field validation measure- ments}

First, a measurement of the acoustic field generated by the source transducer under linear conditions in water was performed to derive a source definition for use in the simulations. For this measurement, the transducer was mounted in the scanning tank on a 2 -axis computer controlled stage $(\theta, \phi)$ and driven with a peak-to-peak drive voltage of $7 \mathrm{~V}$, which was sufficiently low to avoid the generation of harmonics in the field. The transducer beam axis was first aligned with the scanning tank $z$-axis by aligning the hydrophone with the beam axis at two different axial positions. The angular offset of the beam axis relative to the scanning tank axis was then calculated and the angular alignment of the transducer was adjusted accordingly. The process was repeated until the beam axis was aligned with the scanning tank axis to within $0.1^{\circ}$. The pressure was then measured over a $52 \mathrm{~mm}$ by $52 \mathrm{~mm}$ lateral plane at an axial distance of $40 \mathrm{~mm}$ from the origin of the transducer surface, with a step size of $0.4 \mathrm{~mm}$. Lateral scans reaching $20 \mathrm{~mm}$ either side of the beam axis, and axial scans between $z=30 \mathrm{~mm}$ and $z=200 \mathrm{~mm}$, both with a step size of $0.2 \mathrm{~mm}$ and passing through the focus of the field (the position of the maximum voltage squared integral), were also obtained under the same drive conditions for use in validating the source definition before further simulations. The source and measurement configuration is shown in Figure 1. Further axial and lateral scans through the focus were obtained for validation of the simulations under weakly nonlinear conditions in water. These additional line scans were made at five drive levels with peak-to-peak voltages of 13.9, 21.1, 41.9, 63.1 and 73.4 V. For each drive condition, five independent sets of line scans were acquired (the transducer and hydrophone were removed from the tank, remounted and realigned between measurements). At the highest drive level, five harmonics were visible in the spectrum of the focal waveform.

\section{Layered medium configuration and validation measure- ments}

For validating $\mathrm{k}$-Wave simulations in inhomogeneous absorbing media, simple phantoms were constructed in geometric shapes, filled with glycerol and mounted in the scanning tank between the source and hydrophone. Glycerol was chosen as it is reasonably well characterised, has a sound speed approximately $30 \%$ higher than that of water, and has an attenuation coefficient on the same order as soft tissue. The design of these phantoms was intended to cause lensing and refraction of the acoustic field, rather than aberration of the focus, allowing simple comparison of the position and size of the measured and simulated focal regions.

1) Acoustic properties of the medium: The acoustic properties of water, glycerol and Mylar are shown in Table II. The attenuation and sound speed of the glycerol (G7893, ACS reagent $\geqslant 99.5 \%$, Sigma Aldrich Company Ltd., Dorset, UK) was measured as described in Appendix A. The density and nonlinearity parameter were taken from the literature [2123]. The sound speed, density, attenuation coefficient, and nonlinearity parameter of water were obtained as a function of temperature from polynomial fits to the data described in references [24-27] (these have been implemented as functions in k-Wave). The density of Mylar was taken from the manufacturer's datasheet [28]. The sound speed of Mylar was calculated from density, the mean of the elastic moduli in the machine and transverse directions, and the Poisson's ratio, also given on the datasheet.

2) Medium geometry: Two different shaped glycerol filled phantoms were used to introduce medium heterogeneities: a rectangular slab of thickness of $24.7 \mathrm{~mm}$, and a wedge shaped container with thickness increasing from $20 \mathrm{~mm}$ to $40 \mathrm{~mm}$ across its width. Each of the containers was of sufficient lateral width to completely cover the beam area (see Fig. 1). The side, top and bottom walls of the containers were built from 10 $\mathrm{mm}$ thick laser-cut perspex, and the front and back windows (through which the beam passed) were made from $100 \mu \mathrm{m}$ thick Mylar film (Goodfellow Cambridge Ltd., Huntingdon, UK) stretched and glued to the perspex frame. A relatively 
TABLE I

SUMMARY OF PREVIOUS EXPERIMENTAL VALIDATIONS OF MODELS OF ULTRASOUND PROPAGATION.

\begin{tabular}{|c|c|c|c|c|c|c|}
\hline Model & Ref. & Source & Nonlinear & Medium & Heterogeneity & Comparison metrics and agreement \\
\hline k-Wave & [14] & $\begin{array}{l}\text { Broadband } \\
\text { planar (PA) }\end{array}$ & no & water & none & pulse shape: good qualitative agreement \\
\hline k-Wave & [15] & $\begin{array}{l}5 \mathrm{MHz} \\
\text { linear array }\end{array}$ & yes & $\begin{array}{l}\text { water } \\
\text { olive oil }\end{array}$ & $\begin{array}{l}\text { none } \\
\text { none }\end{array}$ & $\begin{array}{l}p_{\text {sptp }}: 12 \% \\
\text { spatial distribution of } p_{f_{0}}, p_{f_{2}}: \text { good qualitative agreement } \\
p_{\text {sptp: }}: 25 \% \\
\text { spatial distribution of } p_{f_{0}}, p_{f_{2}}: \text { good qualitative agreement }\end{array}$ \\
\hline k-Wave & [16] & $\begin{array}{l}1.1 \mathrm{MHz} \\
\text { focusing }\end{array}$ & yes & water & $\begin{array}{l}\text { planar olive oil } \\
\text { wedge olive oil }\end{array}$ & $\begin{array}{l}\text { spatial distribution of } p_{f_{0}}, p_{f_{2}}: \text { good qualitative agreement } \\
\text { spatial distribution of } p_{f_{0}}, p_{f_{2}}: \text { good qualitative agreement }\end{array}$ \\
\hline k-Wave & [17] & $\begin{array}{l}1 \mathrm{MHz} \\
\text { focusing }\end{array}$ & no & water & $\begin{array}{l}\text { skull phantom } \\
\text { araldite } \\
\text { skull phantom } \\
\text { VeroBlack }\end{array}$ & $\begin{array}{l}p_{\text {sptp: }}: 1 \% \\
\text { focal position: } 1 \mathrm{~mm} \\
\text { focal volume: } 2 \% \\
p_{\text {sptp }}: 1 \% \\
\text { focal position: } 1 \mathrm{~mm} \\
\text { focal volume: } 12 \%\end{array}$ \\
\hline $\begin{array}{l}\text { Extended } \\
\text { ASA }\end{array}$ & [19] & $\begin{array}{l}500 \mathrm{kHz} \\
\text { planar* }\end{array}$ & no & water & $\begin{array}{l}\text { incidence angle: } 0-45^{\circ} \\
\text { planar plastic } \\
\text { wedge plastic } \\
\text { planar plastic-rubber- } \\
\text { plastic }\end{array}$ & $\begin{array}{l}\text { results normalised by free field } p_{\text {sptp }} \text {, } \\
\text { on axis amplitude: } 0.05-9 \% \\
\text { mean difference: amplitude } 6-79 \% \text {, phase: } 5.3-30^{\circ} \\
\text { on axis amplitude: } 5.8-32 \% \\
\text { mean difference: amplitude } 8-19 \% \text {, phase: up to } 20^{\circ} \\
\text { on axis amplitude: } 12-68 \% \\
\text { mean difference: amplitude: } 17-57 \% \text {, phase: } 8.7-39^{\circ}\end{array}$ \\
\hline
\end{tabular}

PA $=$ photoacoustic. $p_{\text {sptp }}=$ spatial-peak temporal-peak pressure. $f_{0}$ - fundamental frequency, $f_{2}$ - 2 nd harmonic frequency. ${ }^{*}$ modelled source was different to experimental source.

TABLE II

MEDIUM PROPERTIES USED IN SIMULATIONS, FOR WATER AT $20.6^{\circ} \mathrm{C}$ AND GLYCEROL AT $* 19.1^{\circ} \mathrm{C},{ }^{\dagger} 22^{\circ} \mathrm{C}$ AND $\ddagger 20^{\circ} \mathrm{C}$, AND MYLAR AT ROOM TEMPERATURE.

\begin{tabular}{lccc}
\hline & Water & Glycerol & Mylar \\
\hline Sound speed $\left[\mathrm{m} \mathrm{s}^{-1}\right]$ & 1484 & $1920^{*}$ & 2235 \\
Density $\left[\mathrm{kg} \mathrm{m}^{-3}\right]$ & 998 & $1260^{\dagger}$ & 1390 \\
$\alpha_{0}\left[\mathrm{~dB} \mathrm{~cm}-1 \mathrm{MHz}^{-b}\right]$ & 0.0021 & $0.445^{*}$ & \\
$\mathrm{~b}$ & 2 & $2^{*}$ & \\
B/A & 4.98 & $9.00^{\ddagger}$ & \\
\hline
\end{tabular}

thick Mylar membrane was used in order to ensure the surfaces were planar. When the phantoms were filled, perspex sheets were clamped over the Mylar windows to avoid overfilling and bulging of the surfaces. To obtain a geometrically fixed and well-defined medium configuration, the transducer and phantoms were mounted using optical posts and an optical breadboard fixed above the water tank. The positions of the mounting holes on the breadboard were used to determine the relative positions of the source and phantoms. The front faces of the planar and wedge shaped phantoms were located at distances of $35 \mathrm{~mm}$ and $33.5 \mathrm{~mm}$, respectively, from the source. These source and medium configurations are shown in Fig. 1.

3) Measurement protocol: Alignment of the hydrophone to the beam axis was performed without the phantom in place using time of flight to set the source-to-hydrophone distance. Once the alignment was complete, a phantom was inserted and measurements were made using the original hydrophone coordinates. The acoustic pressure was measured on a $40 \mathrm{~mm}$ by $130 \mathrm{~mm}$ lateral-axial $(x-z)$ plane starting at $z=70$ $\mathrm{mm}$, with step sizes of 0.2 by $0.5 \mathrm{~mm}$. To ensure that the spatial peak pressure was captured by the measurement, the $y$ position of the focus with the phantom in place was located and the plane was measured at that fixed $y$ position. For the measurements made in water, waveforms were acquired in a time window starting before the earliest arrival time of the pulse from any part of the transducer at any measurement 


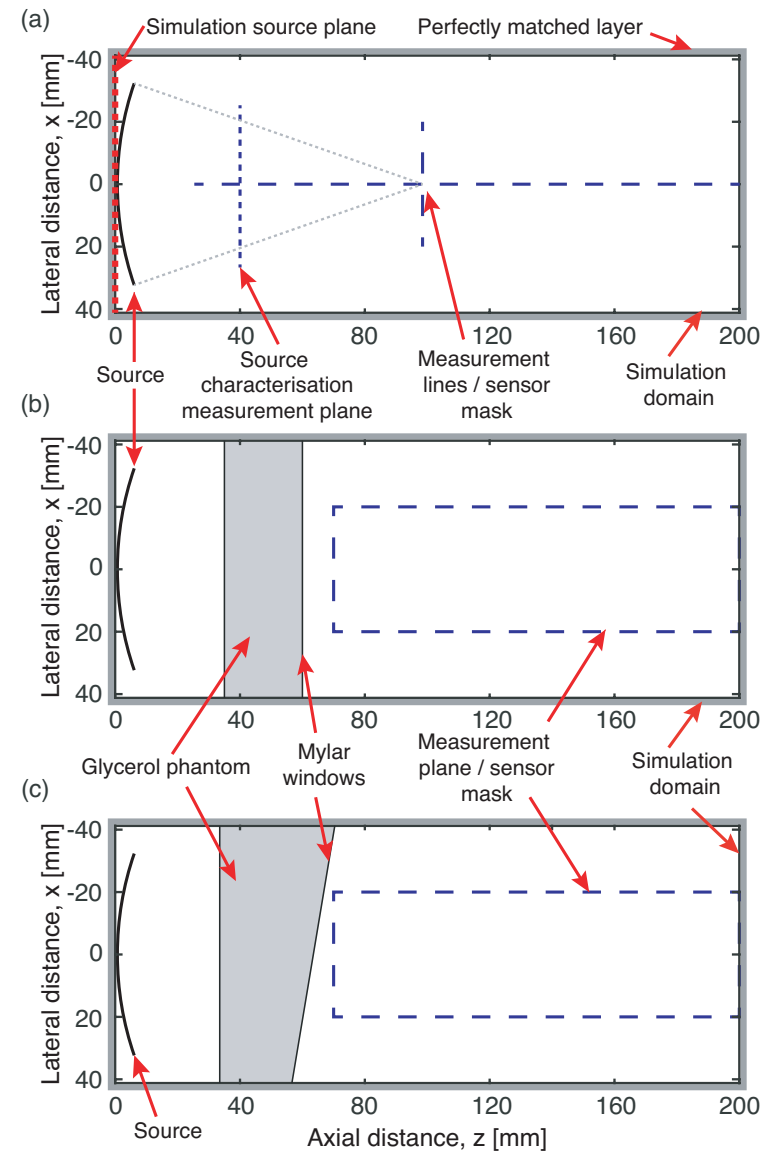

Fig. 1. Schematic diagram of position of the source, fluid medium inclusions, and the planes on which data was measured superimposed on a $x-z$ slice through the 3D simulation domain for (a) free-field conditions, (b) with planar phantom, and (c) wedge shaped phantom.

position, and ending after the arrival of the first reflection from within the phantom. The waveforms were processed as previously described to obtain the acoustic pressure. The peak positive and peak negative pressure, and the magnitudes of the fundamental frequency and first 3 harmonics were extracted and upsampled using Fourier interpolation to match the simulation resolution.

\section{Simulation METHODS}

\section{A. Overview}

Simulations of the acoustic field were performed using version 1.3 of the open-source k-Wave MATLAB toolbox. This solves a system of first-order equations accounting for nonlinearity, medium heterogeneities, and power law absorption, using a k-space pseudospectral method [7]. For all simulations, a k-space correction was also applied to the time varying source to remove the error due to the temporal sampling of the source function [29].

To create an acoustic source in k-Wave, energy was introduced through interior mass sources on a plane coincident with the source origin. The source signals were determined from the planar pressure measurement described in Sec. II-B. The measured signals were downsampled to the temporal resolution required for the simulations and input to a gradient-based optimisation that calculates an equivalent interior mass source that minimises the difference between the $\mathrm{k}$-Wave forward model and the measured data [30]. The resulting source plane was then upsampled using Fourier interpolation to the required simulation spatial resolution. The source amplitude was scaled by the ratio of the drive voltage used during the validation measurements to the drive voltage used during the source characterisation measurements.

\section{B. Simulation parameters and hardware}

All simulations were run on a $2048 \times 864 \times 864$ grid with a spatial step size of $100 \mu \mathrm{m}$, giving a maximum supported frequency of $7.4 \mathrm{MHz}$ (13.5 points-per-wavelength in water at $1.1 \mathrm{MHz}$ ). For simulations in water, the temporal step was 20 ns, giving a Courant-Friedrichs-Lewy (CFL) number of 0.3, and simulations were run for 8603 time steps. For simulations through the layered glycerol media, the temporal step was 15 ns, giving a CFL of 0.23 , and simulations were run for 11467 time steps. To ensure that the simulation parameters had no effect on the simulated pressure, convergence with both spatial and temporal step size was tested for both water and layered media before selection of these discretisation parameters. A perfectly matched layer was imposed on a 20 grid point thick layer at each of the edges of the computational domain. The simulated temporal peak-positive and peak-negative pressure was recorded throughout the computational domain for all simulations. For the water simulations, the time varying pressure was also recorded to match the corresponding axial pressure measurements. The virtual measurement volume covered $11 \times$ $11 \times 1701$ grid points (defined as the sensor mask in k-Wave) to ensure the beam axis was located within the sensor mask. For the glycerol phantom simulations, the sensor mask was set to record the time-varying pressure over a set of consecutive lateral-axial planes covering $401 \times 11 \times 1301$ grid points for the same reason.

The medium properties (sound speed, density, nonlinearity parameter, and power law absorption parameters) were defined in a piecewise homogeneous manner following the geometry shown in Fig. 1. The Mylar membranes were implemented as layers of a single grid point in thickness, with the sound speed and density of Mylar given in Table II. With a spatial sampling of $100 \mu \mathrm{m}$, the error in the 1D transmission coefficient for a water-Mylar-glycerol boundary computed by k-Wave compared to the analytical solution was $0.05 \%$ at $1.1 \mathrm{MHz}$ and $0.75 \%$ at the 2 nd harmonic.

Simulations in water at the lowest and highest drive levels were run using the multi-GPU version of $\mathrm{k}$-Wave on a single compute server (PNY 2U 8 GPU Barebone Server) with 8 NVIDIA Tesla P40 Pascal GPUs each with 3840 CUDA cores and $24 \mathrm{~GB}$ of memory $[8,31]$. The computational domain was divided into 8 sub-domains in an $8,1,1$ configuration, where the first dimension is the direction of propagation, with an overlap size of 8 grid points. The compute time was approximately 1 hour and 10 minutes and the simulations consumed 23.9 GB memory per GPU. Due to resource availability, simulations at other drive levels in water and with the glycerol inclusions (which consumed more memory) were run using the MPI 
version of k-Wave [32] on Salomon, a supercomputer operated by the IT4Innovations National Supercomputing Centre in Ostrava, Czech Republic. The cluster consists of 1008 nodes, each composed of $2 \times$ Intel Xeon E5-2680v3 processors (2.5 $\mathrm{GHz}, 12$ cores) with $128 \mathrm{~GB}$ RAM per node, interconnected by a 7D Enhanced hypercube Infiniband network. Simulations were run on 36 nodes, using a total of 864 cores. The compute time for these simulations was approximately 9.5 hours and the peak memory consumption was $1.3 \mathrm{~TB}$.

\section{Comparison metrics}

There are many possible metrics for comparison of measured and simulated fields, the importance of which depend on the intended application of the model. The comparison metrics used in this study and their sensitivity to differences in the amplitude and spatial distribution of the fields are outlined below:

- spatial-peak temporal-peak positive and negative pressures: this captures the pressure amplitude, but is insensitive to shifts or aberrations of the field;

- maximum difference between the measured and simulated temporal peak positive pressures over the comparison plane ( $L_{\infty}$ error): this is sensitive to spatial shifts and aberrations of the field;

- position of the spatial-peak temporal-peak positive pressure: this captures shifts in the field, but is insensitive to differences in the pressure amplitude and can be insensitive to aberrations;

- $-6 \mathrm{~dB}$ length and width of the focal region: this captures aberrations of the focus.

Further comparisons were made of axial and lateral profiles of the total field and harmonics, and focal waveforms and spectra.

When comparing the measured and modelled pressure, the uncertainties in both the measurements and the model should be considered when deciding if the two are in agreement. Errors in the measured pressure can arise from a variety sources, including variations in the properties of the source and measurement equipment, calibration uncertainty, and processing of the measured data. In our previous work studying the repeated measurement of focused ultrasound fields, it was concluded that broadband fields can be measured with differences of less than $10 \%$ with a range of hydrophones [33]. Consequently, in the current work, simulations and measurements can be said to agree (in terms of pressure amplitude) if the pressures are within the expected measurement uncertainty of $10 \%$. (Note, throughout this paper, the measurement uncertainty is used to refer to this value. It is not based on a formal uncertainty budget, which would be higher given that the uncertainty in hydrophone frequency response can be up to $15 \%$.)

\section{REsults}

\section{A. Free-field validation}

First, to validate the source definition used in simulations, a comparison was made with axial and lateral scans performed under the source characterisation conditions. As an additional comparison, the field was projected from the measured plane
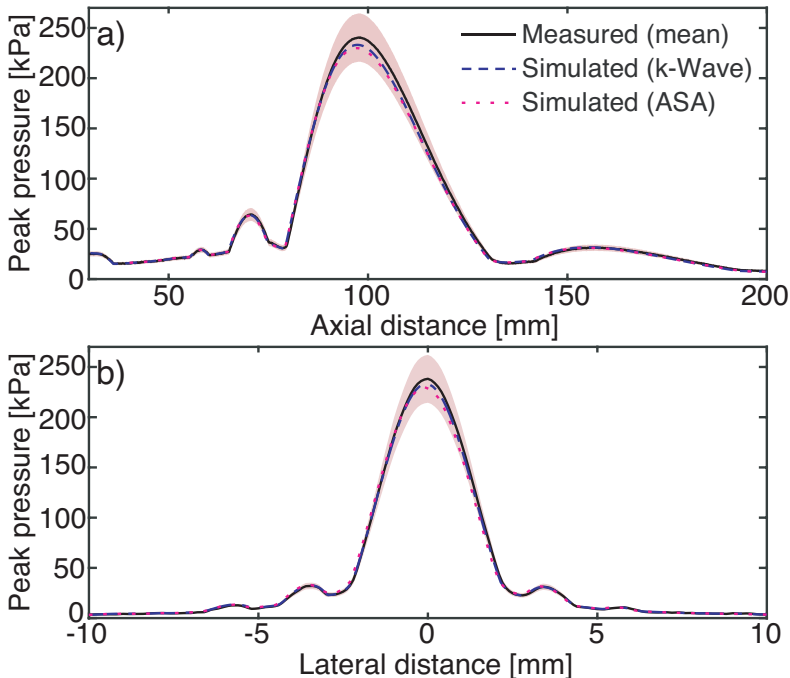

Fig. 2. (a) Axial and (b) lateral spatial peak positive pressure profiles measured and simulated with both $\mathrm{k}$-Wave and with a transient angular spectrum code under linear free-field conditions. The measured profiles show the mean of 5 measurements, the red shading shows $\pm 10 \%$ expected uncertainty on measurements.

using the angular spectrum approach [34]. As shown in Fig. 2 , there is good agreement between the shape and position of the simulated and measured profiles. The simulated pressure is lower in the focal lobe with differences in the spatial-peak positive pressure of $3.0 \%$. The field projected using the angular spectrum approach agrees with $\mathrm{k}$-Wave with a difference in peak focal pressure of $1.4 \%$. This level of agreement is similar to that shown previously with measured data [30] and is within the expected variation on measurements made with the same hydrophone established in our previous work [33]. Both the input plane and the validation measurements were made with the same hydrophone, and since the field is linear, the effect of uncertainty in the hydrophone frequency response should be limited (the short pulse has some bandwidth). It is possible that the differing impact of the directional response of the hydrophone on the input plane and the validation measurements leads to underestimation of the focal pressure [35]; this will be investigated in future work.

Following validation of the source definition, comparisons of the measured and simulated pressures were made at increasing drive levels. The measured and simulated spatial peak-positive and peak-negative pressures and the differences between them are shown in Fig. 3. The differences in the peakpositive pressures ranged from $2.5 \%$ to $6.7 \%$ and from $2.8 \%$ to $9.4 \%$ for the peak-negative pressures. As more energy is moved into the harmonics, there is greater scope for errors to arise in the measured pressure due to uncertainty in the shape and absolute value of the complex frequency response of the hydrophone. However, the differences in the spatial-peak pressures are within $10 \%$ so in this case, the measurements and simulations can be said to agree. For the highest drive level, the comparison metrics described in Sec. III-C are given in Table. III. A comparison of the axial and lateral peak-pressure profiles and the focal waveforms and spectra is shown in Fig. 4. The shape of the measured and simulated profiles agree 


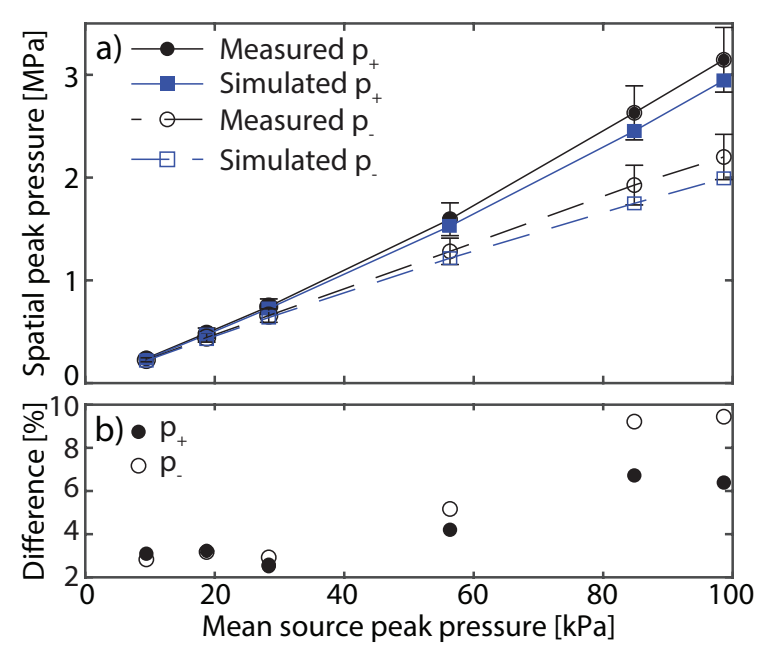

Fig. 3. (a) Mean measured $(n=5)$ and simulated spatial peak positive and peak negative pressures as a function of mean peak positive source pressure, error bars show $10 \%$ uncertainty in the measurement data). (b) The difference between the measured and simulated data.
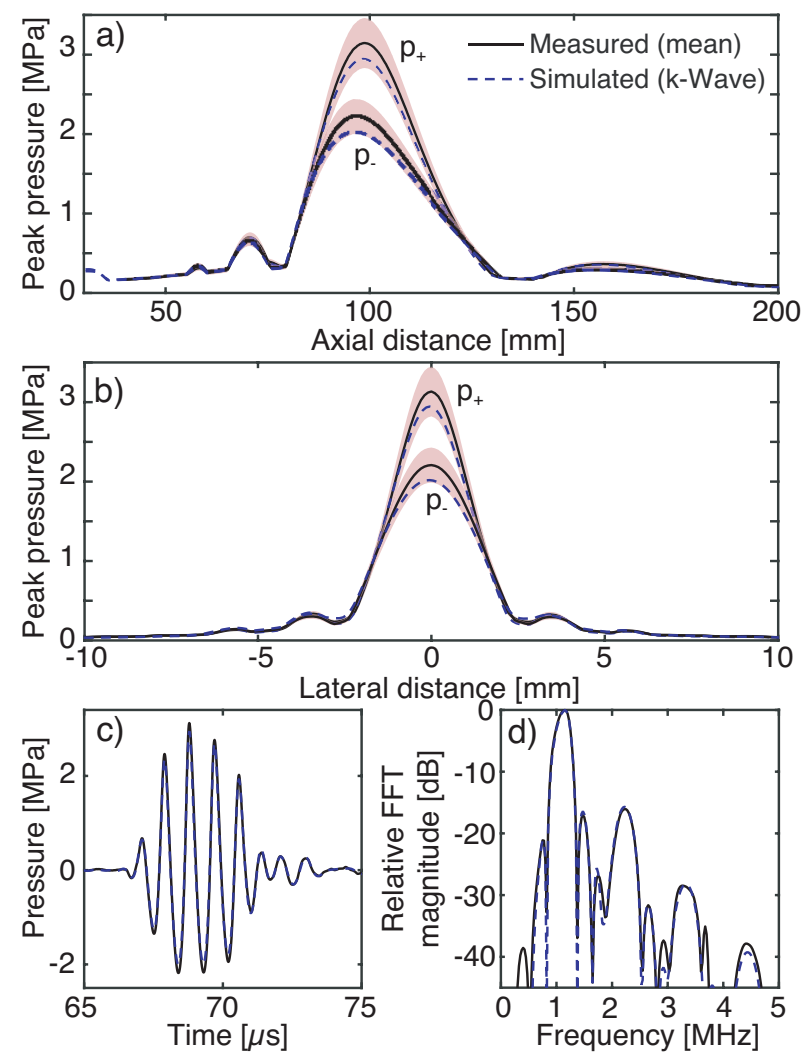

Fig. 4. Measured and simulated field in water at a peak-to-peak drive voltage of 73.4 V. (a) Axial temporal peak pressure profiles. (b) Lateral temporal peak pressure profiles. The measured profiles show the mean of 5 measurements, the red shading shows $\pm 10 \%$ expected uncertainty on measurements. (c) Focal waveforms. (d) Focal spectra.

closely, and the size and position of the focal lobe is similar. The focal waveforms are temporally coincident and similar in shape and the spectra show only small differences, mainly in the amplitude of the fourth harmonic.

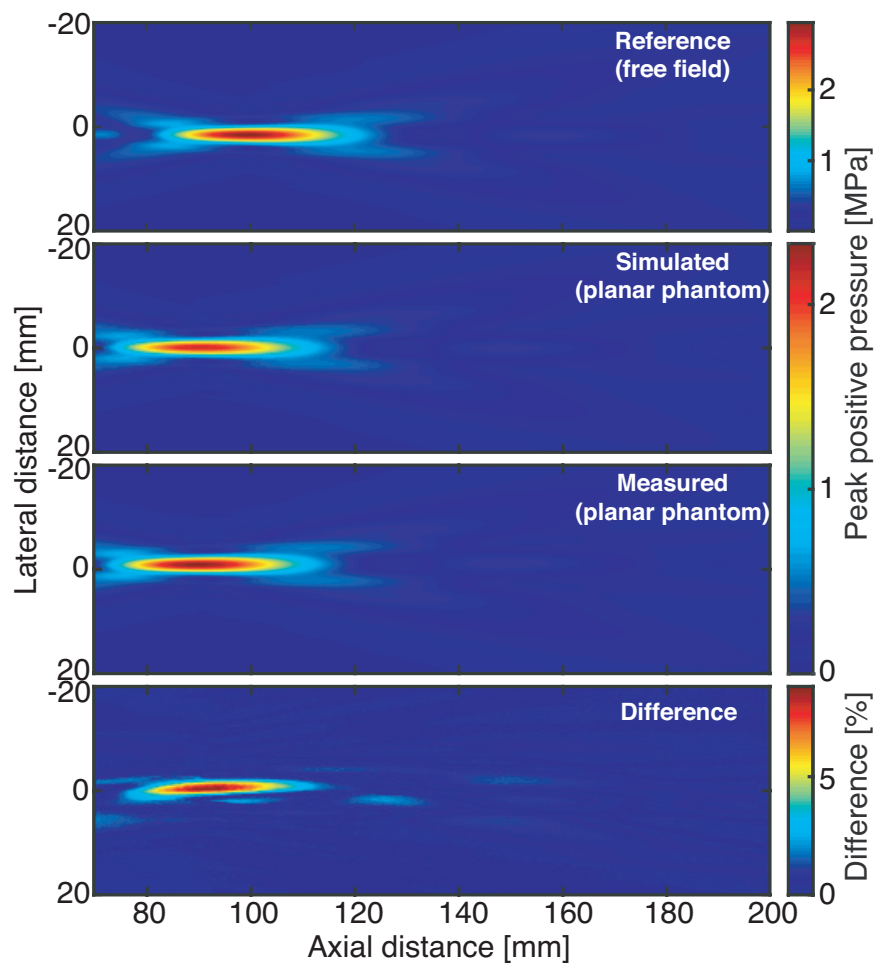

Fig. 5. Simulated and measured spatial peak positive pressures after propagation through the planar glycerol filled phantom and the difference between the measured and simulated data. The corresponding field in water is shown for reference.
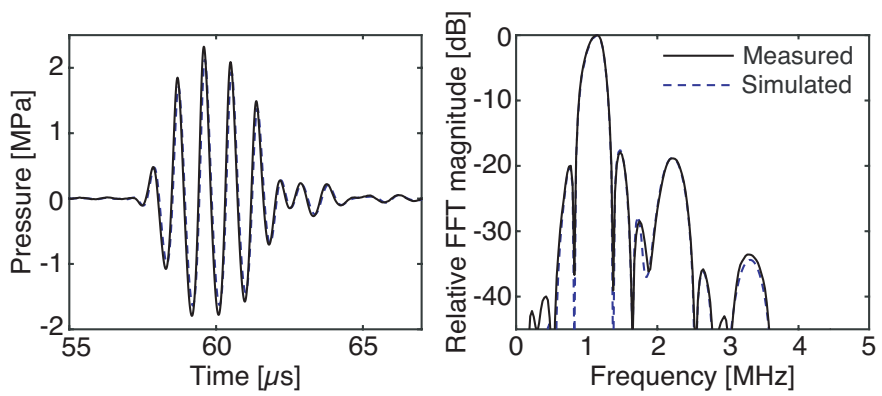

Fig. 6. Simulated and measured spatial peak pressure waveforms and spectra after propagation through the planar glycerol filled phantom.

\section{B. Layered media validation}

The highest drive level used for the water validation was used in the comparison of measurements and simulations with the glycerol phantoms. There is good agreement between the measured and simulated fields in terms of the comparison metrics which are shown in Table III. The presence of the planar glycerol phantom resulted in a decrease in focal pressure of $26 \%(-2.7 \mathrm{~dB})$ compared to the field in water, and a shift in the focal distance of $8 \mathrm{~mm}$. This can be seen in Fig. 5 which shows the measured and simulated pressure on the lateral-axial $(x-z)$ plane after propagation through the planar phantom and the difference between them. The simulated field at the same drive level in water is shown for comparison. The greatest differences are close to the focal region due to slight differences in the position of the focus. The axial position 
TABLE III

COMPARISON OF SPATIAL PEAK PRESSURES AND FOCAL SIZE AND POSITION FOR THE MEASURED AND SIMULATED FIELDS ON THE COMPARISON PLANE AFTER PROPAGATION THROUGH THE PLANAR AND WEDGE SHAPED PHANTOMS.

\begin{tabular}{|c|c|c|c|c|c|c|c|c|c|}
\hline & \multicolumn{3}{|c|}{ Water } & \multicolumn{3}{|c|}{ Planar phantom } & \multicolumn{3}{|c|}{ Wedge phantom } \\
\hline & Measured & Simulated & Diff. & Measured & Simulated & Diff. & Measured & Simulated & Diff. \\
\hline $\operatorname{sptp} p_{+}[\mathrm{MPa}]$ & 3.15 & 2.95 & $6.4 \%$ & 2.32 & 2.13 & $8.5 \%$ & 2.19 & 1.95 & $10.7 \%$ \\
\hline $\operatorname{sptp} p_{-}[\mathrm{MPa}]$ & 2.22 & 2.02 & $9.0 \%$ & 1.80 & 1.65 & $8.3 \%$ & 1.73 & 1.56 & $9.7 \%$ \\
\hline$L_{\infty}$ error in sptp $p_{+}[\%]$ & & & $9.3 \%$ & & & $8.8 \%$ & & & $25.5 \%$ \\
\hline Focal position $(\mathrm{x}, \mathrm{y}, \mathrm{z})[\mathrm{mm}]$ & $0,0,98.5$ & $-0.1,0,98.5$ & $0.1,0,0$ & $-0.1,0.7,90.4$ & $-0.1,0,90.3$ & $0,0.7,0.1$ & $1.4,-0.4,89.7$ & $0.9,0,88.5$ & $0.5,0.4,1.2$ \\
\hline$-6 \mathrm{~dB}$ length of focus $[\mathrm{mm}]$ & 28.6 & 29.3 & 0.7 & 30.3 & 31 & 0.7 & 31.3 & 30.1 & 1.0 \\
\hline$-6 \mathrm{~dB}$ width of focus $[\mathrm{mm}]$ & 2.6 & 2.6 & 0 & 2.8 & 2.8 & 0 & 2.8 & 2.9 & 0.1 \\
\hline
\end{tabular}
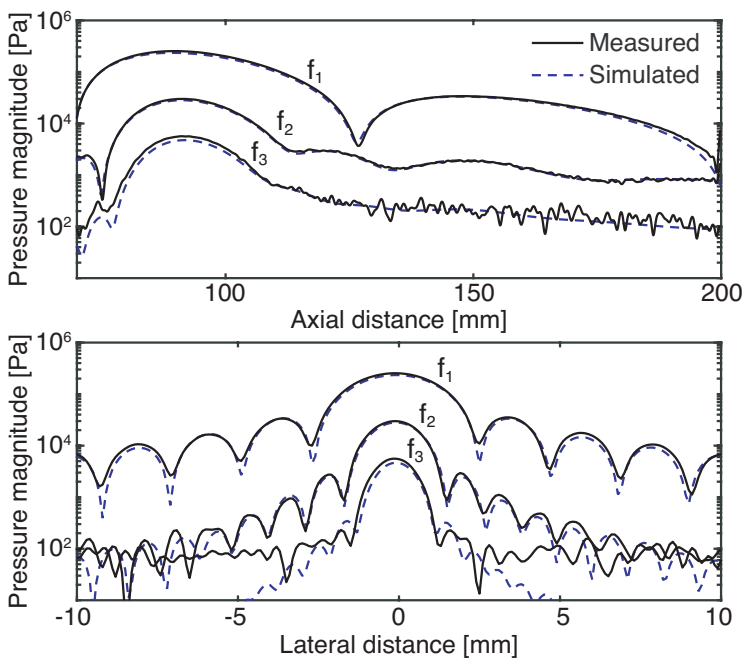

Fig. 7. Simulated and measured axial and lateral profiles of the magnitudes of the first three harmonics after propagation through the planar glycerol filled phantom.

of the focus agrees to $0.1 \mathrm{~mm}$, but there is a shift in the $y$ position in the measurement caused by misalignment or non ideal geometry of the phantom. The focal waveforms and spectra are compared in Fig 6. The spectra and the shape of the waveforms agree closely. Axial and lateral profiles of the first three harmonics for the measured and simulated fields are shown in Fig. 7. There is good agreement between the profiles. The main differences are the deeper nulls in the simulated lateral profiles, and where the measured third harmonic reaches the noise floor.

The presence of the wedge shaped phantom resulted in a decrease in focal pressure of $30 \%(-3 \mathrm{~dB})$ and a shift in focal distance of $8.9 \mathrm{~mm}$ (measured) compared to free-field. This is illustrated in Fig. 8, which shows the simulated free-field alongside the measured and simulated pressure on the $x-z$ plane after propagation through the wedge shaped phantom, and the difference between them. The comparison metrics are shown in Table III. The measured and simulated fields with this phantom also agree well, but in this case there is a larger difference in the measured and simulated focal positions. The field was deflected to a greater extent in the simulation with the beam axis at an angle of $2.3^{\circ}$ to the $z$ axis compared to $1.7^{\circ}$ in the measured field. There is again a shift in the $y$ position of the focus in the measured field. The size of

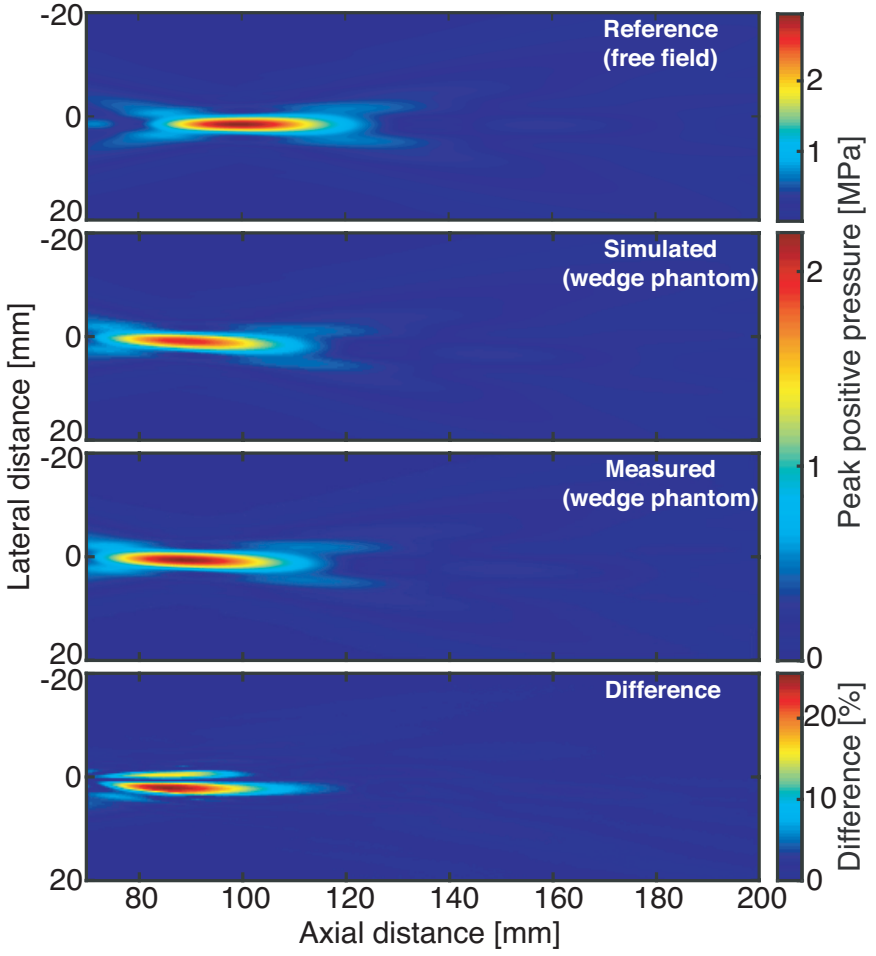

Fig. 8. Simulated and measured spatial peak positive pressures after propagation through the wedge shaped glycerol filled phantom and the difference between the measured and simulated data. The corresponding field in water is shown for reference.
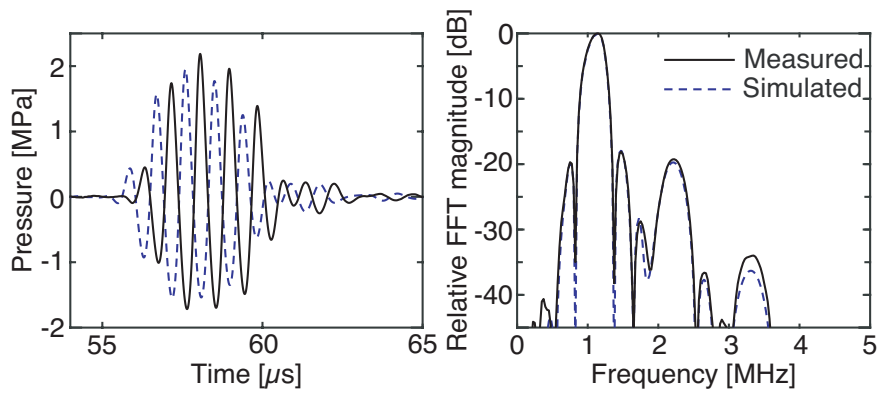

Fig. 9. Simulated and measured spatial peak pressure waveforms and spectra after propagation through the wedge shaped glycerol filled phantom.

the $-6 \mathrm{~dB}$ focal region is similar. The greatest differences are close to the focus due to the difference in angle and position in the measured and simulated fields. Figure 9 shows the focal waveforms and spectra, which again agree well. 

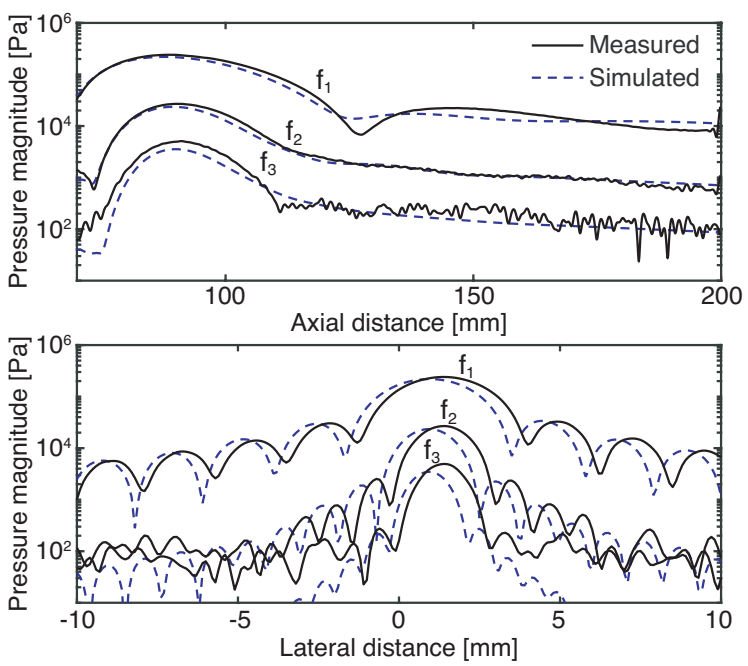

Fig. 10. Simulated and measured axial and lateral profiles of the magnitudes of the first three harmonics after propagation through the wedge shaped glycerol filled phantom.

With this phantom, the simulated waveform arrives before the measured waveform, consistent with the closer focal distance in the simulation. Corresponding differences can be seen in the profiles of the harmonics (see Fig. 10). Due to the difference in the deflection angle of the field, the lateral profiles are clearly offset although their amplitudes agree well, with some difference in the third harmonic which is also visible in the focal spectra. The differences in the beam angle and focal position could indicate a small error in the sound speed of the glycerol or differences in the medium geometry, for example angular or translational misalignment of the phantom with the beam axis, or bulging of the membranes leading to non planar surfaces.

In water and with the planar glycerol phantom, the measured and simulated spatial-peak pressures agree to within our previously established expected measurement uncertainty of $10 \%$. The difference in the spatial-peak positive pressure for the wedge shaped glycerol phantom was slightly larger than this at $10.7 \%$.

In all cases, the simulated pressure is lower than the measured pressure. This could be caused by a systematic error in the hydrophone frequency response or by uncertainty in its frequency dependence. An underestimate of the measured source pressure would propagate to generate larger errors in the simulations of nonlinear fields. Errors could also be introduced in the modelling of the nonlinearity or from incorrect specification of the nonlinearity parameter.

The differences in the spatial-peak pressures with both glycerol phantoms were similar to those observed under freefield conditions, which suggests that the acoustic properties of the glycerol and Mylar membranes have generally been captured and modelled correctly.

A previous sensitivity analysis of simulations of propagation of ultrasound through bone showed that errors in sound speed form the greatest contribution to errors in the amplitude and spatial distribution of pressure, compared to the other acoustic properties [17]. Even small errors were found to have a relatively large influence on focal size and position, for example at $1 \mathrm{MHz}$, a $10 \%$ error in sound speed caused approximately $10 \%$ error in the focal volume. Further errors in both the pressure amplitude and focal position will arise in simulation of the wedge-shaped phantom due to staircasing of the angled side of the glycerol-Mylar-water boundary. These errors have been shown to be on the order of a few \% for the spatial sampling used here [36]. The combination of errors arising from staircasing as well as errors in the angle and position of the boundary are consistent with the larger differences seen between the measured and simulated pressure amplitudes and focal position for the wedge shaped phantom. This highlights the importance of accurate representation of the acoustic properties and geometry of the medium. This poses a significant challenge for simulation of the propagation of ultrasound through media for which the properties are not well known, such as the human body [37].

\section{Previous validations}

The size of the differences reported here are similar to those reported in previous model validation studies which are summarised in Table I. In many cases, the authors report that uncertainty in the medium properties and geometry was a likely cause of discrepancies. However, in one study, the differences were mainly attributed to uncertainty in the frequency response of the needle hydrophone used to make measurements [5]. (It is assumed that given the date of publication, the hydrophone phase sensitivity was not available, and as the frequency response of a similar hydrophone is known to have a nonuniform complex frequency response [33], significant errors in measured pressure are likely [38].) In another study, use of a source definition based on an ideal source introduced differences in the spatial distribution of the measured and simulated fields due to non ideal behaviour of the physical source [18]. In another case, large differences were observed as elastic waves were not modelled but were significant in the measurements [19]. These factors highlight the importance of accurately capturing the source conditions and physics in the model, as well as the challenges of making accurate pressure measurements.

\section{DiscusSiON}

If models of ultrasound propagation are to be used in treatment planning and dosimetry for therapeutic ultrasound, where the position, size, and amplitude of the focus are important, their accuracy must be validated. This can only be done by performing quantitative comparisons. In this work, absolute values of acoustic pressure were compared quantitatively, with no normalisation. The temporally and spatially varying source definition was obtained from measurements, and once obtained, no further corrections were applied. The only scaling of the source pressure was by the drive voltage used during the validation measurements.

Full quantitative validation of models requires that the experimental conditions are replicated as closely as possible in simulation, for example, by the use of holographic measurement-based source definitions and registered medium 
geometries, in addition to careful consideration of the measurement methods. This is important in minimising sources of error not arising from the ability of the model to capture the required physics. Validation of models should then follow an iterative process. Differences between the model and measurement greater than the measurement uncertainty should be investigated so that the underlying causes can be identified and corrections implemented. The process should then be repeated until the model and measurement agree to within the expected measurement uncertainty.

During the course of this validation study, sources of error in both the measurements and simulations were identified and reduced. In terms of the measurements, phase calibrations were obtained for the hydrophones used in our lab and deconvolution of the complex frequency response was implemented to improve the accuracy of pressure measurements, which is especially important for needle hydrophones [38]. We also investigated the expected variation in measurements to quantify how closely we could reasonably expect measurements and models to agree. In terms of the simulations, the way in which the source is defined in $\mathrm{k}$-Wave was modified to reduce errors arising from the use of Dirichlet boundary conditions in pseudospectral time domain solvers [30]. A correction was also implemented to reduce error in time-varying sources [29], and other minor bug fixes have been implemented in new kWave releases. This iterative process will continue in future experimental validation and $\mathrm{k}$-Wave development.

\section{CONCLUSION}

A quantitative validation of the open-source k-Wave toolbox was performed for the nonlinear propagation of ultrasound in layered absorbing fluid media with a measurement based source definition. Under the conditions tested, $\mathrm{k}$-Wave is able to correctly predict fields to close to the expected experimental uncertainty. This demonstrates that acoustic non-linearity and absorption has been modelled correctly, and where the medium properties and geometry are well known and well represented in simulation, the models can be expected to be accurate. This validation will be of use to users of $\mathrm{k}$-Wave and should provide a useful comparison for developers and users of other models wishing to validate the accuracy of acoustic field predictions.

\section{APPENDIX \\ MEASUREMENT OF THE SOUND SPEED AND ATTENUATION COEFFICIENT OF GLYCEROL}

The attenuation coefficient and sound speed of glycerol were measured as a function of frequency using a through transmission substitution method over the frequency range 0.5 to $6 \mathrm{MHz}$ [39]. Glycerol samples were mounted between a planar transducer and large-area membrane hydrophone (30 $\mathrm{mm}$ diameter, GEC-Marconi, UK), which was placed in the far-field. Two transducers were used to cover the frequency range. The first was a $12.7 \mathrm{~mm}$ diameter PZT transducer with a centre frequency of $2.25 \mathrm{MHz}$ driven with a single-cycle pulse to cover the range 0.5 to $3 \mathrm{MHz}$ after propagation through the samples. The second transducer was a $6 \mathrm{~mm}$ diameter PZT transducer with a centre frequency of $15 \mathrm{MHz}$ driven with a

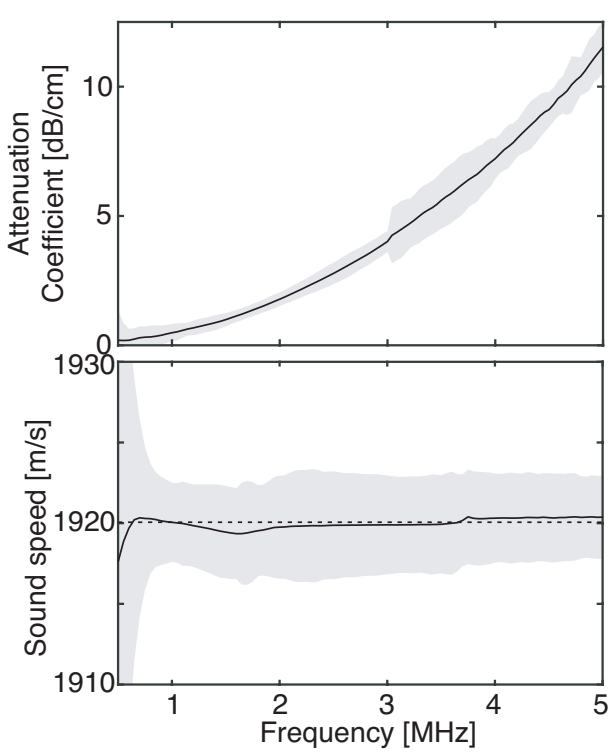

Fig. 11. The measured attenuation coefficient and dispersion of glycerol, the shaded areas show the combined uncertainty in measurements and the dotted line shows the dispersion from the power law fit.

single-cycle pulse to generate signals covering the range 3 to $6 \mathrm{MHz}$ after propagation through the samples. Glycerol was contained in stainless steel sample holders with acoustically transparent $16 \mu \mathrm{m}$ Mylar windows. Two samples of $10.5 \pm$ $0.5 \mathrm{~mm}$ and $25.6 \pm 0.5 \mathrm{~mm}$ thickness (thickness determined from the time of arrival of multiple reflections between the source and sample and within the sample) were used in order to account for interfacial losses at the sample surfaces. During measurements the temperature of the water and glycerol was $19.1^{\circ} \mathrm{C}$.

Waveforms were acquired after propagation through water only and with each of the two samples placed in the beam path. Four sets of measurements were made for each of the frequency ranges at source-hydrophone separations increasing in $1 \mathrm{~cm}$ steps. The frequency-dependent transmission loss for each sample was calculated with respect to the waveforms after propagation through water only. The frequency-dependent attenuation coefficient was then calculated from the difference in transmission loss for the two samples divided by the difference in their thicknesses. The attenuation coefficient followed a power law of the form $\alpha_{0} f^{b}$, where $\alpha_{0}=0.445 \mathrm{~dB} \mathrm{~cm}^{-1}$ $\mathrm{MHz}^{-b}$ and $b=2$. The measured attenuation coefficient as a function of frequency is shown in Fig. 11. The shaded area denotes the combined uncertainties obtained from 3 times the standard deviation of 4 measurement repeats at each frequency, and the uncertainty in the thickness of the samples.

To calculate the speed of sound, the reference (water) waveforms were cross-correlated with the sample waveforms to find the time delay. The delay was then removed to align the waveforms and the residual frequency-dependent phase difference was obtained from the Fourier transforms of the waveforms. The speed of sound as a function of frequency was calculated from the total time delay, the speed of sound in water, and the sample thickness. The measured dispersion and the dispersion calculated from the power law fit to the 
attenuation coefficient are shown in Fig. 11. The mean sound speed over the measured frequency range was $1920 \pm 4 \mathrm{~m}$ $\mathrm{s}^{-1}$, which agrees well with values quoted in the literature $[22,23]$.

\section{REFERENCES}

[1] A. Kyriakou, E. Neufeld, B. Werner, M. M. Paulides, G. Szekely, and N. Kuster, "A review of numerical and experimental compensation techniques for skull-induced phase aberrations in transcranial focused ultrasound," Int. J. Hyperth, vol. 30, no. 1, pp. 36-46, 2014.

[2] V. Suomi, J. Jaros, B. Treeby, and R. O. Cleveland, "Full Modeling of High-Intensity Focused Ultrasound and Thermal Heating in the Kidney Using Realistic Patient Models," IEEE Trans. Biomed. Eng., vol. 65, no. 11, pp. 2660-2670, 2018.

[3] C. Constans, P. Mateo, M. Tanter, and J.-F. Aubry, "Potential impact of thermal effects during ultrasonic neurostimulation: retrospective numerical estimation of temperature elevation in seven rodent setups," Phys. Med. Biol., vol. 63, p. 025003, 2018.

[4] A. D. Pierce, Acoustics: An introduction to its physical principles and applications. Woodbury, N.Y.: Acoustical Society of America, 1989.

[5] R. Williams, E. Cherin, T. Y. J. Lam, J. Tavakkoli, R. J. Zemp, and F. S. Foster, "Nonlinear ultrasound propagation through layered liquid and tissue-equivalent media : computational and experimental results at high frequency," Phys Med Biol., vol. 51, pp. 5809-5824, 2006.

[6] L. Demi, B. E. Treeby, and M. D. Verweij, “Comparison between two different full-wave methods for the computation of nonlinear ultrasound fields in inhomogeneous and attenuating tissue," in 2014 IEEE Int. Ultrason Symp., Sep. 2014, pp. 1464-1467.

[7] B. E. Treeby, J. Jaros, A. P. Rendell, and B. T. Cox, "Modeling nonlinear ultrasound propagation in heterogeneous media with power law absorption using a k-space pseudospectral method," J. Acoust. Soc. Amer., vol. 131, no. 6, p. 4324, 2012.

[8] J. Jaros, F. Vaverka, and B. E. Treeby, "Spectral Domain Decomposition Using Local Fourier Basis : Application to Ultrasound Simulation on a Cluster of GPUs," Supercomput Front Innov, vol. 3, no. 3, pp. 40-55, 2016.

[9] M. Tabei, T. D. Mast, and R. C. Waag, "A k -space method for coupled first-order acoustic propagation equations," J. Acoust. Soc. Amer., vol. 111, no. 53, pp. 53-63, 2002.

[10] N. McDannold, M. Livingstone, C. B. Top, J. Sutton, N. Todd, and N. Vykhodtseva, "Preclinical evaluation of a low-frequency transcranial MRI-guided focused ultrasound system in a primate model," Phys. Med. Biol., vol. 61, pp. 7664-7687, 2016.

[11] C. B. Top, P. J. White, and N. J. McDannold, "Nonthermal ablation of deep brain targets: A simulation study on a large animal model," Med. Phys., vol. 43, no. 2, pp. 870-882, 2016.
[12] W. Legon, P. Bansal, R. Tyshynsky, L. Ai, and J. K. Mueller, "Transcranial focused ultrasound neuromodulation of the human primary motor cortex," Sci Rep., vol. 8, no. 1, pp. 1-14, 2018.

[13] S.-Y. Wu, C. Aurup, C. S. Sanchez, J. Grondin, W. Zheng, H. Kamimura, V. P. Ferrera, and E. E. Konofagou, "Efficient Blood-Brain Barrier Opening in Primates with Neuronavigation-Guided Ultrasound and Real-Time Acoustic Mapping," Sci. Rep., vol. 8, no. 1, p. 7978, 2018.

[14] B. Cox, J. Laufer, K. Koestli, and P. Beard, "Experimental validation of photoacoustic $\mathrm{k}$-space propagation models," Proc. SPIE, vol. 5320, pp. 238-248, 2004.

[15] K. Wang, E. Teoh, J. Jaros, and B. E. Treeby, "Modelling nonlinear ultrasound propagation in absorbing media using the k-Wave toolbox: Experimental validation," IEEE Int.Ultrason. Symp., pp. 523-526, 2012.

[16] E. Martin and B. E. Treeby, "Experimental validation of computational models for large-scale nonlinear ultrasound simulations in heterogeneous, absorbing fluid media," AIP Conf. Proc., vol. 1685, p. 070007, 2015.

[17] J. Robertson, E. Martin, B. Cox, and B. E. Treeby, "Sensitivity of simulated transcranial ultrasound fields to acoustic medium property maps," Phys. Med. Biol., vol. 62, no. 7, pp. 2559-2580, 2017.

[18] Y. Jing and R. O. Cleveland, "Modeling the propagation of nonlinear three-dimensional acoustic beams in inhomogeneous media." J. Acoust. Soc. Amer., vol. 122, no. 3, p. 1352, 2007.

[19] G. T. Clement and K. Hynynen, "Forward Planar Projection Through Layered Media," IEEE Trans. Ultrason., Ferroelectr., Freq. Control, vol. 50, no. 12, pp. 16891698, 2003.

[20] C. J. Vecchio, M. E. Schafer, and P. A. Lewin, "Prediction of ultrasonic field propagation through layered media using the extended angular spectrum method," Ultrasound Med Biol., vol. 20, no. 7, pp. 611-622, 1994.

[21] F. Dong, E. L. Madsen, M. C. MacDonald, and J. A. Zagzebski, "Nonlinearity parameter for tissue-mimicking materials," Ultrasound Med. Biol., vol. 25, no. 5, pp. 831-838, 1999.

[22] M. Sehgal, C. Bahn, and F. Greenleaf, "Measurement of the acoustic nonlinearity parameter B/A in human tissues by a thermodynamic method," J. Acoust. Soc. Amer., vol. 76, no. 4, pp. 1023-1029, 1984.

[23] T. H. Laby and G. W. C. Kaye, "The speed and attenuation of sound," in Tables of Physical \& Chemical Constants, 16th ed., www.kayelaby.npl.co.uk, 2005, ch. 2.4.1.

[24] W. Marczak, "Water as a standard in the measurements of speed of sound in liquids," J. Acoust. Soc. Amer., vol. 102, no. 5, p. 2776, 1997.

[25] F. Jones and G. Harris, "ITS-90 density of water formulation for volumetric standards calibration," J Res Natl Inst Stand Tech, vol. 97, no. 3, p. 335, 1992.

[26] J. M. Pinkerton, "The absorption of ultrasonic waves in liquids and its relation to molecular constitution," Proc. Phys. Soc. Section B, vol. 62, pp. 129-141, 1949. 
[27] R. T. Beyer, "Parameter of Nonlinearity in Fluids," $J$. Acoust. Soc. Amer., vol. 32, no. 6, pp. 719-721, 1960.

[28] "Mylar polyester film - physical-thermal properties," 2003 (accessed 9 July 2019). [Online]. Available: http://usa.dupontteijinfilms.com/wp-content/ uploads/2017/01/Mylar_Physical_Properties.pdf

[29] B. T. Cox and B. E. Treeby, "Accurate time-varying sources in k-space pseudospectral time domain acoustic simulations," in 2018 IEEE Int. Ultrason. Symp., Oct 2018, pp. 1-4.

[30] B. Treeby, F. Lucka, E. Martin, and B. T. Cox, "Equivalent-Source Acoustic Holography for Projecting Measured Ultrasound Fields through Complex Media," IEEE Trans. Ultrason., Ferroelectr., Freq. Control, vol. 65, no. 10, pp. 1857-1864, 2018.

[31] B. Treeby, F. Vaverka, and J. Jaros, "Performance and accuracy analysis of nonlinear k-Wave simulations using local domain decomposition with an 8-GPU server," Proc Meet Acoust, vol. 34, p. 022002, 2018.

[32] J. Jaros, A. P. Rendell, and B. E. Treeby, "Full-wave nonlinear ultrasound simulation on distributed clusters with applications in high-intensity focused ultrasound," Int. J. High Perform. Comput. Appl., vol. 30, no. 2, pp. 137-155, 2016.

[33] E. Martin and B. Treeby, "Investigation of the repeatability and reproducibility of hydrophone measurements of medical ultrasound fields," J. Acoust. Soc. Amer., vol. 145, no. 3, pp. 1270-1282, 2019.

[34] X. Zeng and R. J. McGough, " Evaluation of the angular spectrum approach for simulations of near-field pressures," J. Acoust. Soc. Amer., vol. 123, no. 1, pp. 68-76, 2008.

[35] M. A. Ghanem, A. D. Maxwell, W. Kreider, B. W. Cunitz, V. A. Khokhlova, O. A. Sapozhnikov, and M. R. Bailey, "Field Characterization and Compensation of Vibrational Nonuniformity for a 256-Element Focused Ultrasound Phased Array," IEEE Trans. Ultrason., Ferroelectr., Freq. Control, vol. 65, no. 9, pp. 1618-1630, 2018.

[36] J. Robertson, B. Cox, J. Jaros, and B. E. Treeby, "Accurate simulation of transcranial ultrasound propagation for ultrasonic neuromodulation and stimulation," J. Acoust. Soc. Amer, vol. 141, no. 3, pp. 1726-1738, 2017.

[37] B. E. Treeby, J. Jaros, E. Martin, and B. T. Cox, "From biology to bytes: Predicting the path of ultrasound waves through the human body," Acoustics Today, vol. 15, no. 2, pp. 36-44, 2019.

[38] K. A. Wear, P. M. Gammell, S. Maruvada, Y. Liu, and G. R. Harris, "Improved measurement of acoustic output using complex deconvolution of hydrophone sensitivity," IEEE Trans. Ultrason., Ferroelectr., Freq. Control, vol. 61, no. 1, pp. 62-75, 2014.

[39] B. Zeqiri, W. Scholl, and S. P. Robinson, "Measurement and testing of the acoustic properties of materials: a review," Metrologia, vol. 47, no. 2, pp. S156-S171, 2010. 